\title{
Uncommon CD markers in acute myeloid leukemia
}

\author{
Abul Kalam Azad, Md. Rafiquzzaman Khan, A. B. M. Hassan Habib and Masuda Begum
}

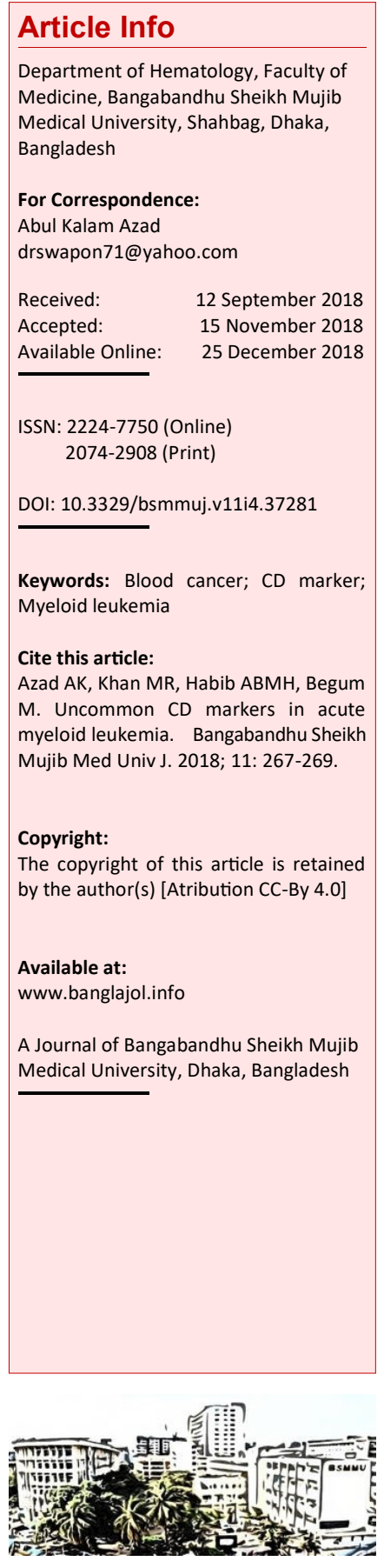

\section{Abstract}

This study was done to assess the unusual CD expression in 100 cases of acute myeloid leukemia from October 2016 to April 2018. The age limit was from 3 to 50 years. Four color flow cytometry was used to diagnose the fresh aspirated bone marrow or peripheral blood sample of acute leukemia. The unusual lymphoid CD expression on myeloblasts was analyzed. Among the cases, $44 \%$ were acute myeloid leukemia, $52 \%$ of patients were of acute lymphoblastic leukemia and mixed phenotype acute leukemia was $4 \%$. Aberrant CD expression was observed in $58 \%$ acute myeloid leukemia patients. Both aberrant CD5 and CD7 lymphoid markers expressed in acute myeloid leukemia patients were $4.2 \%$. Aberrant CD7, CD5, cCD79a and cCD3 were in 45.8\%, $33.3 \%, 8.3 \%, 8.3 \%$ of acute myeloid leukemia patients respectively. In acute myeloid leukemia, the frequency of aberrant $\mathrm{CD}$ expression was compared with recent international data.

\section{Introduction}

Blood cancer is $6.5 \%$ of all cancer worldwide, 1 but higher in the Western countries than in Asia and African. WHO predicts that the hematological cancer patients would rise about $48 \%$ in underdeveloped countries by $2030 . \underline{2-4}$ Acute myeloid and lymphoblastic leukemia are $42.4 \%$ of hematological cancer and the frequency of acute myeloid leukemia was $28.3 \%$

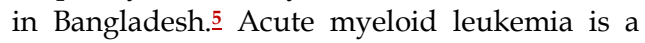
hematological malignancy with morphologic, immunologic, and molecular features with different surface and cytoplasmic CD antigens expression. The CD markers on white blood cells can be determined by flow cytometry. Flow cytometry improves both closeness and reproducibility of acute leukemia classification. 6 Flow cytometry of acute myeloid leukemia has shown variable antigens expressed on stem cells with infrequent co-expression of lymphoid antigens. Acute myeloid leukemia generally exhibits aberrant antigen expression or coexpression in relation to normal myeloid cells. Detection of aberrant phenotypes by flow cytometry has been applied toward monitoring of residual disease. 7

Acute leukemia diagnosis depends on the morphology, cytochemistry and immunophenotyping. The study of aberrant CD expression in acute myeloid leukemia was observed in different parts of the world.

\section{Materials and Methods}

\section{Sample collection}

Consecutive 100 acute leukemia patients aged 3 to 50 years were studied. Three milliliter peripheral blood or $5 \mathrm{~mL}$ of bone marrow aspirated samples were taken in vials containing ethylenediamine tetraacetate (EDTA).

\section{Sample processing}

Using Sysmex XE-2100, the complete blood count was done and blood film was stained by Leishman's stain to find out the presence of blast cells. 8 After the evaluation of peripheral and bone marrow smear of blast cells, cell lysing and fixing method for immunofluorescence staining, the whole blood or aspirate marrow samples were prepared with different $\mathrm{CD}$ markers which were conjugated with fluorochromes (i.e., FITC, PE, ECD and PC5) and with phosphate buffer saline $\left(\mathrm{NaH}_{2} \mathrm{PO}_{4} \cdot 2 \mathrm{H}_{2} \mathrm{O}, \mathrm{Na}_{2} \mathrm{HPO}_{4}\right.$ and $\left.\mathrm{NaCl}\right)$ cell washing was done. The monoclonal antibody was added to the whole blood or aspirated marrow sample, the fluorochrome-labeled antibodies bind specifically to leucocyte surface antigens and/or cytoplasmic antigens. The stained samples were treated with optilyse C lysine solution which lysed the erythrocytes under hypotonic conditions.

The solution containing 15\% formaldehyde and $50 \%$ diethylene glycol used for cytoplasmic 


\begin{tabular}{|lc|}
\hline \multicolumn{2}{|c|}{ Table I } \\
\hline \multicolumn{2}{|c|}{ Distribution of acute leukemia } \\
\hline Cases & $\begin{array}{c}\text { Number of } \\
\text { patients }\end{array}$ \\
\hline Age distribution (Years) & \\
<20 & 52 \\
>20 & 48 \\
Acute myeloid leukemia & 44 \\
Acute myeloid leukemia with conventional CD expres- & 20 \\
sion & 24 \\
Acute myeloid leukemia with aberrant CD expression of & \\
lymphoid markers & 2 \\
cCD79a & 8 \\
CD5 & 2 \\
cCD3 & 11 \\
CD7 & 1 \\
CD5 and CD7 & 52 \\
Acute lymphoblastic leukemia & 4 \\
Mixed phenotype acute leukemia & 100 \\
Total number of acute leukemia & \\
\hline
\end{tabular}

staining of antigens such as MPO, CD79a, CD3, TdT and $\mathrm{CCD} 22$. Sodium azide was used in immunofluorescent as a biotinylated primary labeling reagents. Cell fix was used for the fixation of peripheral blood cell suspension after immunofluorescence staining with monoclonal antibodies. Before flow cytometric analysis, a buffered solution containing less than $10 \%$ formaldehyde and $1 \%$ sodium azide was used. These samples were acquired in the flow cytometry instrument using CXP software.?

\section{Data analysis}

Data were analyzed using software CXP. The fluorescence intensities of the blasts were compared with the negative cell population for different $C D$ marker expressions. The blasts were identified by low side scatter (SSC) and weak CD45 intensity. $\underline{10}$

\section{Results}

Among this study population, acute myeloid leukemia was $44 \%$, acute lymphoblastic leukemia was $52 \%$ and mixed phenotype acute leukemia $4 \%$ (Table I). Among the acute leukemia patients, 24\% cases show aberrant expressions of CD antigens and which was $54.5 \%$ of the total acute myeloid leukemia patients.

Among the 44 acute myeloid leukemia patients, 20 case having conventional CD antigen expressions of lineage-specific markers and it was $20 \%$ of the total acute leukemia patients and $45.5 \%$ of acute myeloid leukemia which were not lineage specific markers.

cCD79a showed aberrancy in 2 out of 24 aberrant acute myeloid leukemia patients which were $8.3 \%$ of total aberrant acute myeloid leukemia. CD5 showed aberrancy in 8 acute myeloid leukemia which was $33.3 \%$ of the total aberrant acute myeloid leukemia. cCD3 showed aberrancy on 2 acute myeloid leukemia which was $8.3 \%$ of the total aberrant acute myeloid leukemia.

CD7 showed aberrancy in 11 acute myeloid leukemia which were $45.8 \%$ of the total aberrant. Aberrancy CD5 and CD7 showed $4.2 \%$ of total aberrant acute myeloid leukemia.

\section{Discussion}

The uncommon lymphoid antigens expression in acute myeloid leukemia were analyzed. In this study, acute myeloid leukemia aberrant cases were more than conventional ones. The mean age was higher in non-lineage specific cases than that of the conventional one.

Acute lymphoblastic leukemia cases were slightly higher than acute myeloid leukemia probably majority patients were younger than 20 years. Among aberrant cases CD7 and CD5 were predominant.

In this study, lymphoid antigens expression in acute myeloid leukemia was 54.5\%. Venditti et al. (1998) reported the frequency of lymphoid markers in acute myeloid leukemia were $41 \%$ which was lower than this study. 8 Weir and Borowitz (2001) observed the frequency of aberrant lymphoid CD expression in acute myeloid leukemia was 13 to $60 \%$ which is close to this study.9Sarma et al. (2015) also stated that aberrant lymphoid CD expression found in $58.3 \%$ of cases of acute myeloid leukemia. 10 Reading et al. (1993) 11 and Abdulateef et al. (2014) $\underline{12}$ reported that a higher incidence of lymphoid markers expression in acute myeloid leukemia was $54.0 \%$ and $67.5 \%$, respectively, which was similar to this study.

The aberrant lymphoid CD7 is the most frequently expressed in acute myeloid leukemia was $45.8 \%$ in our study. Macedo et al. (1995) $\underline{13}$ reported CD7 expression on acute myeloid leukemia was $37.5 \%$; Juluis et al. (2005) $\underline{14}$ reported $32.6 \%$ and Orgata et al. (2001) $\underline{15}$ stated $30.9 \%$ CD7 expression on acute myeloid leukemia. Weir and Borowitz (2001) 9 and Auewarakul et al. (2003) 16 stated that the T-cell associated antigen CD7 was seen in $27 \%$.

In this study, $\mathrm{T}$ cell-associated aberrant CD5 expression in acute myeloid leukemia was $33.3 \%$.

Khurram et al. (2010) 17 stated the frequency of aberrant expression of CD5 on acute myeloid leukemia in between $20 \%$ to $40 \%$ of cases of acute 
myeloid leukemia, which is similar to this study. Launder et al. (1996) $\underline{18}$ reported the expression of aberrant CD5 on acute myeloid leukemia was $25 \%$, which was lower than this study.

Variation in study result may be due to different sample size, single or multicenter, different genetic and environmental factors.

\section{Conclusion}

Aberrant $\mathrm{CD}$ is present in the majority of acute myeloid leukemia patients admitted into the Bangabandhu Sheikh Mujib Medical University Hospital.

\section{References}

1. Zhu H, Niu T, Meng W, Xu C, Lei S. Immunophenotype of acute leukemia and its clinical significance. Hua Xi Yi Ke Da Xue Bao. 2002; 33: 118-20.

2. Das P, Horton R. Bangladesh: Innovating for health. Lancet 2013; 382: 1681-82.

3. Hussain SA, Sullivan R. Cancer control in Bangladesh. Jpn J Clin Oncol. 2013; 43: 1159-69.

4. Story HL, Love RR, Salim R, Roberto AJ, Krieger $\mathrm{JL}$, Ginsburg OM. Improving outcomes from breast cancer in a low-income country: Lessons from Bangladesh. Int J Breast Cancer. 2012; 2012.

5. Hossain MS, Iqbal MS, Khan MA, Rabbani MG, Khatun H, Munira S, Miah M, Kabir AL, Islam N, Dipta TF, Rahman F. Diagnosed hematological malignancies in Bangladesh: A retrospective analysis of over 5000 cases from 10 specialized hospitals. BMC Cancer. 2014; 2014.

6. Lewis RE, Cruse JM, Sanders CM, Webb RN, Suggs JL. Aberrant expression of T-cell markers in acute myeloid leukemia. Exp Mol Pathol. 2007; 83: 462-63.

7. Buccisano F, Maurillo L, Del Principe MI, Del Poeta G, Sconocchia G, Lo-Coco F, Arcese W, Amadori S, Venditti A. Prognostic and therapeutic implications of minimal residual disease detection in acute myeloid leukemia. Blood 2012; 119: 332-41.

8. Venditti A, Del Poeta G, Buccisano F, Tamburini A, Cox-Froncillo MC, Aronica G, Bruno A, Del Moro B, Epiceno AM, Battaglia A, Forte L. Prognostic relevance of the expression of TDT and CD7 in 335 cases of acute myeloid leukemia. Leukemia 1998; 12: 1056-63.

9. Weir EG, Borowitz MJ. Flow cytometry in the diagnosis of acute leukemia. Semin Hematol. 2001; 38: 124-38.

10. Sarma A, Hazarika M, Das D, Kumar Rai A, Sharma JD, Bhuyan C, Kataki AC. Expression of aberrant $\mathrm{CD}$ markers in acute leukemia: A study of 100 cases with immunophenotyping by multiparameter flow cytometry. Cancer Biomarkers. 2015; 15: 501-05.

11. Reading $\mathrm{CL}$, Estey EH, Huh YO, Claxton DF, Sanchez G, Terstappen LW, O'Brien MC, Baron S, Deisseroth AB. Expression of unusual immunophenotype combinations in acute myelogenous leukemia. Blood 1993; 81: 3083-90.

12. Abdulateef NA, Ismail MM, Aljedani H. Clinical significance of co-expression of aberrant antigens in acute leukemia: A retrospective cohort study in Makah Al Mukaramah, Saudi Arabia. Asian Pac J Cancer Prev. 2014; 15: 221-27.

13. Macedo A, Orfao A, Vidriales MB, Lopez-Berges MC, Valverde B, Gonzalez M, Caballero MD, Ramos F, Martinez M, Fernandez-Calvo J, Martinez A. Characterization of aberrant phenotypes in acute myeloblastic leukemia. Ann Hematol. 1995; 70: 189-94.

14. Juluis MC, Robert EL, Samuel P, John L. Aberrant expression of CD7, CD56, CD79a antigens in acute myeloid leukemia. Exp Mol Pathol. 2005, 79: 3941.

15. Ogata K, Yokose N, Shioi Y, Ishida Y, Tomiyama J, Hamaguchi H, Yagasaki F, Bessyo M, Sakamaki H, Dan K, Kuriya S. Reappraisal of the clinical significance of CD7 expression in association with cytogenetics in de novo acute myeloid leukaemia. Br J Haematol. 2001; 115: 612-15.

16. Auewarakul CU, Promsuwicha OY, U-pratya Y, Pattanapanyasat K, Issaragrisil S. Immunophenotypic profile of adult acute myeloid leukemia (AML): Analysis of 267 cases in Thailand. Asian Pac J Allergy Immunol. 2003; 21: 153-60.

17. Khurram MM, Jafri SA, Mannan A, Nadeem A, Jamal A. Frequency of aberrant expression of CD markers in cases of acute leukemia. Med J Islamic World Acad Sci. 2010; 18: 55-60.

18. Launder TM, Bray RA, Stempora L, Chenggis ML, Farhi DC. Lymphoid-associated antigen expression by acute myeloid leukemia. Am J Clin Pathol. 1996; 106: 185-91. 